\title{
Osteopontin controls immunosuppression in the tumor microenvironment
}

\author{
Michael R. Shurin \\ Departments of Pathology and Immunology, University of Pittsburgh Medical Center, Pittsburgh, Pennsylvania, USA.
}

\begin{abstract}
Cancer cells evade the immune system through a variety of different mechanisms, including the inhibition of antitumor effector T cells via checkpoint ligand-receptor interaction. Moreover, studies have shown that blocking these checkpoint pathways can reinvigorate the antitumor immunity, thereby prompting the development of numerous checkpoint immunotherapies, several of which are now being approved to treat multiple types of cancer. However, only a fraction of patients achieves promising long-term outcomes in response to checkpoint inhibition, suggesting the existence of additional unknown tumor-induced immunosuppressive pathways. In this issue of the $J C I$, Klement and colleagues describe an additional pathway of T cell inhibition in cancer. Specifically, the authors demonstrate that downregulation of IRF8, a molecular determinant of apoptotic resistance, in tumor cells aborts repression of osteopontin, which in turn binds to its physiological receptor CD44 on activated T cells and suppresses their activation. These results suggest that osteopontin may act as another immune checkpoint and may serve as a target to expand the number of patients who respond to immune checkpoint inhibitor therapy.
\end{abstract}

\section{How do tumors escape} immunological surveillance?

The immune system plays a crucial role in destroying cancerous cells. However, cancer cells also evolve and gain the ability to escape immunological elimination. Mechanisms that support evasion of immune attack include the selection of tumor variants that are considered invisible to immune cells, such as those with low MHC or chemokine expression, that can suppress activity and/or function of immune cells, that produce IL-10, TGF- $\beta$, gangliosides, galectins, prostaglandins, or indoleamine 2,3-dioxygenase (IDO), etc., or those that can polarize antitumor immune cells into a protumorigenic phenotype. For instance, tumor expression of coinhibitory molecules like programmed cell death protein 1 ligand 1 (PD-L1, also known as B7 homolog 1 [B7H1] and CD274) may inhibit $\mathrm{T}$ cell activation, induce $\mathrm{T}$ cell exhaustion, alter cytokine production, and cause apoptosis and anergy of effector $\mathrm{T}$ cells, which is attributable to tumor growth and progression (1).

Immune checkpoint blockade has been demonstrated to be a promising method for cancer treatment and is currently being investigated in more than 1,000 clinical trials (2). Application of immune checkpoint inhibitors in clinical practice has had a groundbreaking effect on cancer treatment. However, reliable responses and promising long-term outcomes are observed in only a fraction of patients. Primary refractoriness and acquired resistance after achieving objective response are major problems limiting the effectiveness checkpoint blocking therapy (3). Mechanisms that underlie these failures are poorly understood, but

Related Article: p. 5549

Conflict of interest: The author has declared that no conflict of interest exists.

Reference information: / Clin Invest. 2018;128(12):5209-5212. https://doi.org/10.1172/JCI124918. 


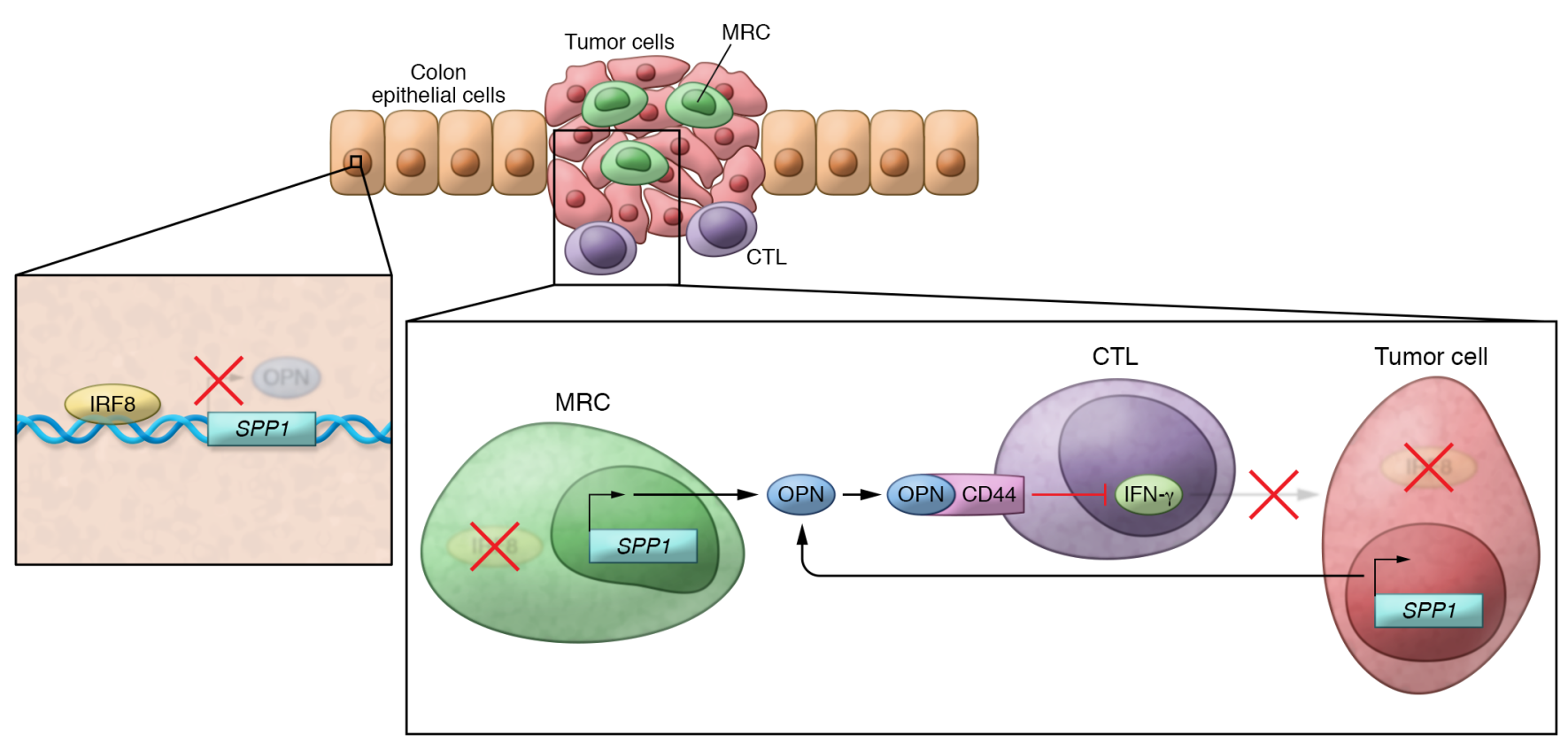

Figure 1. IRF8-controlled expression of osteopontin in the tumor microenvironment suppresses activity of $\mathrm{T}$ cells and supports tumor progression. Osteopontin (OPN) has diverse biological functions in many physiological and pathological processes. Via its cytokine, chemotactic, and cell signaling functions, OPN regulates inflammation, biomineralization, tissue remodeling, and angiogenesis. In the tumor microenvironment, OPN facilitates cell-matrix interactions and promotes tumor progression. In this issue of the $J C I$, Klement et al. demonstrate that OPN may also suppress activity of cytotoxic CD8 ${ }^{+}$ T lymphocytes (CTLs), thereby contributing to progression of malignant disease. OPN is highly expressed in myeloid regulatory cells (MRCs) and malignant cells, two major components of the tumor microenvironment, and suppresses T cell proliferation and IFN- $\gamma$ secretion by binding to CD44, which is highly expressed on activated T Iymphocytes. IRF8 may act as a tumor suppressor, as it represses OPN (encoded by SPP1) expression in normal epithelial cells. Nevertheless, IRF8 expression is silenced and OPN expression is elevated during transformation of epithelial cells into a malignant phenotype. Together, this suggests that tumor cells and myeloid regulatory cells may exploit downregulation of IRF8 to upregulate OPN as a mechanism to suppress CD8 ${ }^{+}$ T cell-mediated antitumor immunity.

sis, and rheumatoid arthritis, whereas OPN-deficient mice are protected against rheumatoid arthritis, autoimmune hepatitis, multiple sclerosis, intestinal bowel disease, type 1 diabetes mellitus, and Sjögren's syndrome (5). Though the immunomodulatory properties of OPN have been proven in different models of autoimmune disease, the involvement of these properties in immune regulation in the tumor microenvironment is still incompletely understood.

In this issue, Klement and colleagues provide new insights into mechanisms of OPN-induced T cell suppression (10). Specifically, the authors demonstrate that OPN is highly expressed in myeloidderived suppressor cells with polymorphonuclear morphology (PMN-MDSCs) and tumor cells, two major components of the tumor microenvironment, and can directly suppress proliferation and IFN- $\gamma$ production by $\mathrm{CD} 8^{+} \mathrm{T}$ cells, the primary effectors responsible for cancer immunosurveillance and elimination of malignant cells. Importantly, Klement et al. have revealed a pre- viously uncharacterized tumor suppressor function of IRF8, by showing that IRF8 binds to the Spp1 promoter region to repress OPN expression in colon epithelial cells (10). However, during the transformation of colon epithelial cells into colon tumor cells, IRF8 expression is silenced and OPN is elevated, suggesting that tumor cells may use downregulation of IRF8 to upregulate OPN as a mechanism to suppress $\mathrm{CD} 8^{+} \mathrm{T}$ cellmediated antitumor immunity (Figure 1).

\section{IRF8-OPN-CD44 axis in cancer}

IRF8 is a transcription factor that regulates hematopoiesis, and disruption of its activity may promote leukemogenesis. IRF8 is constitutively expressed in monocytes, dendritic cells, and B cells, and it regulates maturation of microglia cells. In solid tumors, following induction by IFN- $\gamma$, IRF8 sensitizes carcinoma cells to Fas-mediated apoptosis (11). Aberrant methylation of the IRF8 gene in solid tumors has also been detected and is considered a molecular determinant of apoptotic resistance and a metastatic phenotype (12). IRF8 has also been shown to be induced in T cells following antigen stimulation, but its functions in this situation are less well understood. Klement et al. demonstrated that IRF8 deficiency leads to abnormal generation of antigen-specific $\mathrm{CD} 8^{+} \mathrm{T}$ cells in vivo and results in an increased number of $\mathrm{CD} 8^{+} \mathrm{T}$ cells expressing high levels of CD44 (10).

CD44 is a multifunctional transmembrane glycoprotein that was first identified as a receptor for hyaluronan and later shown to bind several other ligands, including OPN, collagen, fibronectin, chondroitin, and matrix metalloproteinases (13). CD44 is broadly expressed and is vital in the regulation of hyaluronic acid metabolism, wound healing, and cytokine release, and in the activation of lymphocytes, monocytes, and keratinocytes (14). One or multiple splice variants and standard CD44 may be expressed in malignant cells and regulate migration, proliferation, and survival, as well as stimulating intratumoral angiogenesis (15).

Klement et al. report that splenocytes from Irf ${ }^{--}$mice express levels of OPN that 
are more than 10 times greater than those in cells from WT animals. Moreover, 95\% of OPN-expressing cells in the spleen of IRF8-deficient mice were PMN-MDSCs, and as OPN is a natural ligand for CD44, it can inhibit $\mathrm{T}$ cell activation and IFN- $\gamma$ production in vitro (10). Along with the demonstration that mice with $\mathrm{T}$ cell-specific IRF8 deficiency display a normal response to immunization, these data suggest that IRF8 regulates $\mathrm{CD}^{+} \mathrm{T}$ cell activation through a cell-extrinsic mechanism via OPNexpressing MDSCs. Consistent with what was observed in PMN-MDSCs, Klement et al. determined that IRF8 expression is significantly downregulated, whereas OPN expression is significantly upregulated, in colon cancer tissues as compared with the normal colon in vivo (10).

Thus, there is the molecular link between myeloid cells and $\mathrm{T}$ cells in the context of IRF8 function and $\mathrm{T}$ cell activation. Specifically, IRF8 acts as a repressor of OPN expression in myeloid cells to facilitate $\mathrm{T}$ cell activation under physiological conditions. In the tumor milieu, OPN is highly expressed in PMN-MDSCs and malignant cells in the absence of IRF8, and thereby suppresses CD $44^{+} \mathrm{T}$ cells. In other words, MDSC- and tumor-derived OPN may function as an inhibitory ligand that negatively regulates $\mathrm{T}$ cell activation in the tumor microenvironment to promote cancer progression.

\section{Conclusions and future studies}

OPN may be highly expressed in MDSCs and malignant cells, two major components of the tumor microenvironment, and thus represents an alternative target for cancer therapy due to its role in regulating multiple survival signaling pathways. Moreover, the data by Klement et al. support this notion and show that OPN may also inhibit activation of cytotoxic T lymphocytes (10), such that OPN functions as an alternative immune checkpoint ligand. However, because OPN is a multifaceted agent that exerts a cell-specific role in inflammation, immunity, and tissue repair, simply neutralizing its activity in different pathological conditions likely will not be an appropriate or feasible approach.

The heterogeneous activities of OPN may be ascribed to multiple variants, including those that result from transcriptional, posttranscriptional, and posttrans- lational modifications, such as phosphorylation, proteolytic cleavage, sialylation, and transglutaminase crosslinking (16). These OPN variants are differentially involved in the pathogenesis of diseases, and understanding the role of individual variants is critical to adapt appropriate therapeutic approaches to target OPN. Within the tumor milieu, OPN may display complex activities, as it is expressed by both malignant and stromal cells, both of which also have receptors for OPN. Thus, whether tumor-derived OPN differs structurally or functionally from stromal-derived OPN remains to be clarified. In addition, preferential expression of various isoforms of CD44 in certain tissues has been observed, with the appearance of some CD44 isoforms associated with inhibition of tumor metastasis (17).

Klement et al. also report that serum OPN levels are markedly higher in patients with colon cancer than in healthy donors and inversely correlate with patient survival (10). Interestingly, OPN levels are also significantly higher in patients with rheumatoid arthritis compared with patients with osteoarthritis, elevated in patients with Crohn's disease, and may predict major adverse cardiovascular events $(18,19)$. OPN is a promising biomarker of earlystage melanoma and oral squamous cell carcinoma $(20,21)$. However, the presence of different isoforms can only partly account for the multiplicity of functions ascribed to OPN. Another important aspect is the ability of OPN to interact with different receptors in addition to CD44. For instance, OPN contains RGD and SVVYGLR sequences, which interact with integrins (22). Therefore, OPN can chemoattract macrophages and neutrophils (9). Because of its highly anionic nature (almost one-third of its 300 amino acids are negatively charged), OPN may also bind damage-associated molecular pattern molecules, an activity that likely plays a role in preventing tissue injury and regulating inflammation. With this new information presented by Klement et al., the next step will be to develop criteria for expanding the number of selected patients that will respond, and respond well, to agents that prevent immune checkpoint signaling.

Address correspondence to: Michael R. Shurin, Immunopathology, UPMC, Clini- cal Lab Building, Room 4024, 3477 Euler Way, Pittsburgh, Pennsylvania 15213, USA. Phone: 412.647.6140; Email: shurinmr@ upmc.edu.

1. Sun C, Mezzadra R, Schumacher TN. Regulation and function of the PD-L1 checkpoint. Immunity. 2018;48(3):434-452.

2. Chen L, Han X. Anti-PD-1/PD-L1 therapy of human cancer: past, present, and future. J Clin Invest. 2015;125(9):3384-3391.

3. Ribas A, Wolchok JD. Cancer immunotherapy using checkpoint blockade. Science. 2018;359(6382):1350-1355.

4. Romero D. Immunotherapy: PD-1 says goodbye, TIM-3 says hello. Nat Rev Clin Oncol. 2016;13(4):202-203.

5. Clemente $\mathrm{N}$, et al. Osteopontin bridging innate and adaptive immunity in autoimmune diseases. J Immunol Res. 2016;2016:7675437.

6. Denhardt DT, Noda M, O'Regan AW, Pavlin D, Berman JS. Osteopontin as a means to cope with environmental insults: regulation of inflammation, tissue remodeling, and cell survival. J Clin Invest. 2001;107(9):1055-1061.

7. Rangaswami H, Bulbule A, Kundu GC. Osteopontin: role in cell signaling and cancer progression. Trends Cell Biol. 2006;16(2):79-87.

8. Patarca R, et al. Structural and functional studies of the early T lymphocyte activation 1 (Eta-1) gene. Definition of a novel T cell-dependent response associated with genetic resistance to bacterial infection. JExp Med.1989;170(1):145-161.

9. Morimoto J, Kon S, Matsui Y, Uede T. Osteopontin; as a target molecule for the treatment of inflammatory diseases. Curr Drug Targets. 2010;11(4):494-505.

10. Klement JD, et al. An osteopontin/CD44 immune checkpoint controls $\mathrm{CD} 8^{+} \mathrm{T}$ cell activation and tumor immune evasion. JClin Invest. 2018;128(12):5549-5560.

11. Yang D, et al. IFN regulatory factor 8 mediates apoptosis in nonhemopoietic tumor cells via regulation of Fas expression. J Immunol. 2007;179(7):4775-4782

12. Suzuki M, et al. Aberrant methylation and silencing of IRF8 expression in non-small cell lung cancer. Oncol Lett. 2014;8(3):1025-1030.

13. Shirasaki T, et al. The osteopontin-CD44 axis in hepatic cancer stem cells regulates IFN signaling and HCV replication. Sci Rep 2018;8(1):13143.

14. Chen C, Zhao S, Karnad A, Freeman JW. The biology and role of CD44 in cancer progression: therapeutic implications. J Hematol Oncol. 2018;11(1):64.

15. Wang SJ, Wong G, de Heer AM, Xia W, Bourguignon LY. CD44 variant isoforms in head and neck squamous cell carcinoma progression. Laryngoscope. 2009;119(8):1518-1530.

16. Gao X, et al. Osteopontin alters DNA methylation through up-regulating DNMT1 and sensitizes $\mathrm{CD} 133^{+} / \mathrm{CD} 44^{+}$cancer stem cells to 5 azacytidine in hepatocellular carcinoma. J Exp Clin Cancer Res. 2018;37(1):179.

17. Ouhtit A, et al. TGF- $\beta 2$ : a novel target of CD44-promoted breast cancer invasion. J Cancer. 2013;4(7):566-572. 
18. Carbone F, et al. Serum levels of osteopontin predict major adverse cardiovascular events in patients with severe carotid artery stenosis. Int $J$ Cardiol. 2018;255:195-199.

19. Liu LN, et al. Circulating levels of osteoprotegerin, osteocalcin and osteopontin in patients with rheumatoid arthritis: a systematic review and meta-analysis [published online ahead of print September 6, 2018]. Immunol Invest. https:// doi.org/10.1080/08820139.2018.1510957.

20. D’Addazio G, Artese L, Traini T, Rubini C, Caputi S, Sinjari B. Immunohistochemical study of osteopontin in oral squamous cell carcinoma allied to fractal dimension. J Biol Regul Homeost Agents. 2018;32(4):1033-1038.

21. Treskova I, et al. OPG, OPN, EGF and VEGF levels at individual Breslow score stages in malignant melanoma. Anticancer Res. 2018;38(8):4907-4911.

22. Sharif SA, et al. Thrombin-activatable carboxypeptidase $\mathrm{B}$ cleavage of osteopontin regulates neutrophil survival and synoviocyte binding in rheumatoid arthritis. Arthritis Rheum. 2009;60(10):2902-2912. 